\title{
Clinicopathologic and molecular features of sporadic early-onset colorectal adenocarcinoma: an adenocarcinoma with frequent signet ring cell differentiation, rectal and sigmoid involvement, and adverse morphologic features
}

Daniel T Chang ${ }^{1, *}$, Rish K Pai ${ }^{2, *}$, Lisa A Rybicki ${ }^{3}$, Michael A Dimaio ${ }^{4}$, Maneesha Limaye ${ }^{1}$, Priya Jayachandran ${ }^{1}$, Albert C Koong ${ }^{1}$, Pamela A Kunz ${ }^{5}$, George A Fisher ${ }^{5}$, James M Ford ${ }^{5}$, Mark Welton ${ }^{6}$, Andrew Shelton ${ }^{6}$, Lisa $\mathrm{Ma}^{4}$, Daniel A Arber ${ }^{4}$ and Reetesh K Pai ${ }^{4}$

${ }^{1}$ Department of Radiation Oncology, Stanford University, Stanford, CA, USA; ${ }^{2}$ Department of Pathology, Cleveland Clinic, Cleveland, OH, USA; ${ }^{3}$ Department of Quantitative Health Sciences, Cleveland Clinic, Cleveland, OH, USA; ${ }^{4}$ Department of Pathology, Stanford University, Stanford, CA, USA; ${ }^{5}$ Department of Medicine/Oncology, Stanford University, Stanford, CA, USA and ${ }^{6}$ Department of Surgery, Stanford

University, Stanford, CA, USA

Recent literature suggests an increasing incidence of colorectal carcinoma in young patients. We performed a histologic, molecular, and immunophenotypic analysis of patients with sporadic early-onset ( $\leq \mathbf{4 0}$ years of age) colorectal carcinoma seen at our institution from the years 2000-2010 and compared these tumors to a cohort of consecutively resected colorectal carcinomas seen in patients $>40$ years of age. A total of 1160 primary colorectal adenocarcinomas were surgically resected for the years 2000 through 2010 . Of these, $75(6 \%)$ were diagnoses in patients $\leq \mathbf{4 0}$ years of age of which $13(17 \%)$ demonstrated abnormalities in DNA mismatch repair, $4 \mathbf{( 5 \% )}$ were in patients with known germline genetic disorders (two patients with familial adenomatous polyposis, one patient with juvenile polyposis, and one patient with Li-Fraumeni syndrome), and three patients (4\%) had long-standing chronic inflammatory bowel disease. The sporadic early-onset colorectal carcinoma group comprised a total of 55 patients $(55 / 1160,5 \%)$ and were compared with a control group comprising 73 consecutively resected colorectal carcinomas with proficient DNA mismatch repair in patients $>40$ years of age. For the early-onset colorectal carcinoma group, most cases $(33 / 55,60 \%)$ were diagnosed between the age of $\mathbf{3 5}$ and $\mathbf{4 0}$ years of age. Compared with the control group, the early-onset colorectal carcinoma group was significantly different with respect to tumor location $(P<0.007)$ with $80 \%(44 / 55$ cases) identified in either the sigmoid colon $(24 / 55,44 \%)$ or rectum $(20 / 55,36 \%)$. Morphologically, early-onset colorectal carcinomas more frequently displayed adverse histologic features compared with the control colorectal carcinoma group such as signet ring cell differentiation $(7 / 55,13 \%$ vs $1 / 73,1 \%, P=0.021)$, perineural invasion $(16 / 55,29 \%$ vs $8 / 73,11 \%$, $P=0.009)$ and venous invasion $(12 / 55,22 \%$ vs $4 / 73,6 \%, P=0.006)$. A precursor adenomatous lesion was less frequently identified in the early-onset colorectal carcinoma group compared with the control group (19/55, 35\% vs $39 / 73,53 \%, P=0.034)$. Of the early-onset colorectal carcinomas, only $2 / 45$ cases (4\%) demonstrated $K R A S$ mutations compared with $11 / 73(15 \%)$ of the control group colorectal adenocarcinomas harboring KRAS mutations, although this difference did not reach statistical significance $(P=0.13)$. BRAF V600E mutations were not identified in the early-onset colorectal carcinoma group. No difference was identified between the two

Correspondence: Dr RK Pai, MD, Department of Pathology, Stanford University, 300 Pasteur Drive, Room H-2110, Stanford, CA 94305, USA.

E-mail: rpai75@gmail.com

${ }^{*}$ These two authors are co-first authors.

Received 29 December 2011; revised 23 February 2012; accepted 24 February 2012; published online 6 April 2012 
groups with regard to tumor stage, tumor size, number of lymph node metastases, lymphatic invasion, tumor budding, mucinous histology, or tumor-infiltrating lymphocytes. Both groups had similar recurrence-free $(P=0.28)$ and overall survival $(P=0.73)$. However, patients in the early-onset colorectal carcinoma group more frequently either presented with or developed metastatic disease during their disease course compared with the control colorectal carcinoma group $(25 / 55,45 \%$ vs $18 / 73,25 \%, P=0.014)$. In addition, $8 / 55$ patients $(15 \%)$ in the early-onset colorectal carcinoma group developed local recurrence of their tumor while no patients in the control colorectal carcinoma group developed local recurrence $(P<0.001)$, likely due to the increased incidence of rectal carcinoma in the patients with early-onset colorectal carcinoma. Our study demonstrates that colorectal carcinoma is not infrequently diagnosed in patients $\leq \mathbf{4 0}$ years of age and is not frequently the result of underlying Lynch syndrome or associated with other cancer-predisposing genetic conditions or chronic inflammatory conditions. These tumors have a striking predilection for the distal colon, particularly the sigmoid colon and rectum and are much more likely to demonstrate adverse histologic factors, including signet ring cell differentiation, venous invasion, and perineural invasion.

Modern Pathology (2012) 25, 1128-1139; doi:10.1038/modpathol.2012.61; published online 6 April 2012

Keywords: colorectal carcinoma; early-onset; signet ring

Colorectal carcinoma is the second most common cause of cancer-related death in the United States. ${ }^{1}$ Although colorectal carcinoma is generally thought to be a disease that affects the elderly, recent literature suggests an increasing incidence of colorectal carci-

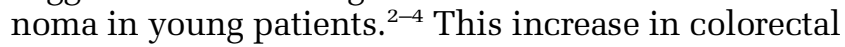
carcinoma in young patients has been observed despite a decreased overall incidence and mortality of colorectal carcinoma possibly due to preventive cancer screening in older patient populations. ${ }^{2,4}$ The increased incidence of colorectal carcinoma in young patients appears to be limited to the rectum and rectosigmoid. ${ }^{2,3}$ Data from the Surveillance, Epidemiology, and End Results registry (SEER, 1973-2005) identified an increasing annual percentage change of $2.2 \%$ of rectosigmoid cancer diagnosed in patients under the age of 40 years. ${ }^{2}$ The greatest percentage change in rectal carcinoma incidence was identified in patients between 35 and 39 years. ${ }^{2}$ The increasing incidence of rectal carcinoma in young patients is important to recognize as the diagnosis of colorectal carcinoma in young patients is often not clinically suspected and such patients may be incorrectly managed early in their disease course.

The limited literature on early-onset colorectal carcinoma reveals a wide range of reported clinical outcomes for young patients with colorectal carcinoma. A number of reports have demonstrated poor clinical outcomes for these patients. ${ }^{5-9}$ Other reports have found an equivalent or better cancer-specific survival rates in young patients compared with older patients despite presenting with advanced stage disease. ${ }^{8,10}$ The limited literature data are also conflicting with regards to the more common locations of colorectal carcinoma, with some indicating increased frequency of proximal right-sided colorectal carcinoma ${ }^{11}$ while others demonstrating increased frequency of rectal and rectosigmoid carcinoma. $^{3,12}$ Most of the published data are confounded by inclusion of hereditary colorectal carcinoma, particularly Lynch syndrome-associated colorectal carcinomas, which have differing genetic events leading to tumorigenesis and often have improved survival compared with non-syndromic colorectal carcinoma. ${ }^{13,14}$

We performed a histologic, molecular, and immunophenotypic analysis of patients with sporadic early-onset ( $\leq 40$ years of age) colorectal carcinoma seen at our institution from the years 2000-2010 and compared the tumors to a cohort of consecutively resected colorectal carcinomas with available pathologic material seen in patients $>40$ years of age. Our results demonstrate that colorectal carcinoma is not infrequently diagnosed in patients $\leq 40$ years of age and is not often the result of underlying Lynch syndrome or associated with other cancer-predisposing genetic conditions or chronic inflammatory conditions. These tumors have a striking predilection for the distal colon, particularly the sigmoid colon and rectum, and are much more likely to demonstrate adverse histologic factors, including signet ring cell differentiation, venous invasion, and perineural invasion. However, overall and recurrence-free survival in young patients with colorectal carcinoma does not appear to be reduced.

\section{Materials and methods}

\section{Study Group}

The clinicopathologic records of patients with primary invasive adenocarcinoma of the colon or rectum diagnosed before or at the age of 40 and accessioned at the Department of Pathology at Stanford University Hospital for the years 20002010 were reviewed. A control group of colorectal adenocarcinomas consisted of consecutively resected colorectal adenocarcinoma diagnosed after the age of 40 years seen at Stanford University Hospital for the years 2005 and 2006 with available pathologic material. Specifically excluded from this study were neuroendocrine tumors, neuroendocrine 
carcinomas, squamous cell carcinomas, and adenocarcinomas of the appendix. The pathology reports and hospital charts were reviewed and the following information was obtained: age, gender, presence of risk factors including colorectal polyposis syndrome and inflammatory bowel disease, type of initial surgical procedure, and the anatomic site of tumor at initial presentation. Intraoperative and clinical follow-up data were obtained from hospital and clinic charts under the guidelines of the Stanford University Institutional Review Board.

\section{Pathologic Evaluation}

All available cases of colorectal adenocarcinoma diagnosed in patients $\leq 40$ years of age and control group colorectal adenocarcinomas were histologically reviewed and the following histologic features were recorded for each tumor: grade, extent of invasion, lymph node metastases, status of margin of resection, lymphatic invasion, perineural invasion, venous invasion, tumor budding, tumor-infiltrating lymphocytes, Crohn's-like peritumoral reaction, presence and type of precursor lesion, and synchronous colorectal polyps. The grade of each colonic adenocarcinoma was scored using a two-tiered scheme: low grade, $>50 \%$ gland formation and high grade, $<50 \%$ gland formation. Tumor budding assessment was performed using the rapid bud count method: ${ }^{15}$ high budding, $>50 \%$ of areas examined under $\times 200$ magnification were positive for budding and low budding, $<50 \%$ of areas examined under $\times 200$ magnification were positive for budding. The presence of tumor-infiltrating lymphocytes was defined as $>7$ lymphocytes per 10 high-powered fields. ${ }^{16}$ Crohn's-like peritumoral reaction was scored as absent, mild, or intense, as previously described. ${ }^{17}$ The presence or absence of precursor polyps and the histologic subtype of the precursor polyp associated with invasive colonic adenocarcinoma was recorded. ${ }^{18,19}$ For patients with rectal adenocarcinomas receiving neoadjuvant therapy, the pretreatment biopsy samples were also reviewed. Recurrences, if any, were also histologically confirmed when available.

\section{Immunohistochemical Analysis}

Mismatch repair protein immunohistochemistry was performed using the standard streptavidin-biotinperoxidase procedure. Primary monoclonal antibodies against MLH1 (clone G168-728, BD PharMingen, San Diego, CA, USA, 1:200), MSH2 (clone FE11, Oncogene Research Products, Cambridge, MA, USA, 1:100), MSH6 (clone 44, BD Transduction, San Jose, CA, USA, 1:200), and PMS2 (clone MRQ-28, Cell Marque, Rocklin, CA, USA, 1:10) were applied to 4- $\mu$ m-thick formalin-fixed, paraffin-embedded sections. The sections were deparaffinized in xylene, and rehydrated through graded alcohols to distilled water before undergoing antigen retrieval by heat treatment in either citrate solution pH 6.0 (MLH1, PMS2, and MSH2) or EDTA solution pH 9.0 (MSH6). An automated detection using a Leica Bond Autostainer (Buffalo Groove, IL, USA) was employed. Normal expression was defined as nuclear staining within tumor cells, using infiltrating lymphocytes as positive internal control. Negative protein expression was defined as complete absence of nuclear staining within tumor cells in the face of concurrent positive labeling in internal non-neoplastic tissues.

Immunohistochemical stains were performed on 4- $\mu$ m-thick sections of paraffin-embedded tissue from each case with the following commercially available antibodies, dilutions, and antigen retrieval conditions: p16 ${ }^{\mathrm{INK} 4 \mathrm{~A}}$ (clone E6H4, CINtec, EDTA pretreatment, prediluted, Leica Bond autostainer), E-cadherin (clone 4A2C7, Invitrogen, EDTA pretreatment, 1:25 dilution, Leica Bond autostainer), and $\beta$-catenin (clone 14, Cell Marque, EDTA pretreatment, 1:50 dilution, Ventana XT autostainer (Tucson, AZ, USA)). Human papilloma virus in-situ hybridization (HPV ISH) was performed using prediluted Ventana reagents after pretreatment with citrate ( $\mathrm{pH}$ 6.0) and protease on a Ventana XT autostainer using a I'View plus detection kit. All slides were reviewed by a single pathologist (RKP) blinded to treatment outcomes. The labeling was scored for extent of staining. For $\mathrm{p} 16^{\mathrm{INK} 4 \mathrm{~A}}$ nuclear and/or nuclear and cytoplasmic staining in $>10 \%$ of tumor cells was considered positive. For E-cadherin, membranous staining in $>50 \%$ of tumor cells was considered positive. For $\beta$-catenin, nuclear labeling in $>50 \%$ of tumor cells was considered positive. For p16 ${ }^{\text {INK4A }}$ and HPV ISH, a paraffin-embedded section of invasive uterine cervical squamous cell carcinoma was included as a positive control for each run. For $\beta$-catenin, paraffin-embedded tissue sections of known-positive fibromatosis were included as a positive control for each run. In addition, negative control serum was applied to all control sections as a negative control.

\section{Microsatellite Instability Analysis Polymerase Chain Reaction}

Forty-two colorectal carcinomas were also analyzed using the ProMega MSI analysis system utilizing a panel of five mononucleotide microsatellite markers (BAT-25, BAT-26, NR-21, NR-24, and MONO-27) and two pentanucleotide repeats (Penta C and Penta D) incorporated into a multiplex fluorescence assay. ${ }^{20}$ Briefly, the tests were performed on tumor and normal DNA extracted from paraffin-embedded tissue blocks using a DNease Tissue Kit (Qiagen, Valencia, CA, USA); manual microdissection was performed, when required, to exclude overabundance of non-lesional tissue. Paired DNA samples from neoplastic and non-neoplastic samples were genotyped and analyzed by capillary electrophoresis using an ABI 310 Genetic Analyzer (Applied 
Biosystems, Foster City, CA, USA). In accordance with NCI guidelines, microsatellite instability at 2 loci or more was defined as MSI-high (MSI-H), instability at a single locus was defined as MSI-low (MSI-L), and no instability at any of the loci tested was defined as microsatellite stable (MSS).

\section{BRAF and KRAS Mutation Analysis}

DNA was extracted from paraffin sections, using the DNease Blood and Tissue Kit (Qiagen) after xylene deparaffinization and ethanol wash. Mutant KRAS was detected using a validated KRAS mutation kit (Entrogen, Tarzana, CA, USA) that identifies the common somatic mutations located in codons 12, 13, and 61 of the KRAS gene. The mutations were detected on an Applied Biosystems 7900 (Applied Biosystems). The evaluation of KRAS assay results was performed according to the manufacturer's instructions.

$B R A F$ mutation analysis at codon 600 (V600E) was performed by a real-time PCR based on an allelic discrimination method previously described. ${ }^{21,22}$ Briefly, real-time PCR was performed using allelespecific primers designed to selectively amplify the wild-type (T1796) and mutant (A1796) BRAF alleles. The primer sequences were as follows: $\mathrm{V}$, 5'-GTGATTTTGGTC TAGC TACtGT; E, 5'-CGCGG CCGGCCGCGGCGGTGATTTTGGTCTA GCTACcGA; and AS, 5'-TAGCCTCAATTCTTACCAT CCAC. PCR amplification and melting curve analysis were performed on an iCycler iQ (Bio-Rad, Hercules, CA, USA). Genomic DNA was amplified in a $25-\mu$ l volume containing $1 \times$ Platinum SYBR Green qPCR SuperMix-UDG (Invitrogen, Carlsbad, CA, USA), forward primer $V(300 \mathrm{nM})$, forward primer $\mathrm{E}$ $(900 \mathrm{nM})$ and reverse primer AS $(300 \mathrm{nM})$. The cycling conditions were as follows: $50{ }^{\circ} \mathrm{C}$ for $2 \mathrm{~min}$, $95^{\circ} \mathrm{C}$ for $2 \mathrm{~min}, 40$ cycles of $95^{\circ} \mathrm{C}$ for $15 \mathrm{~s}$, and $60^{\circ} \mathrm{C}$ for $60 \mathrm{~s}$. After amplification, samples were subjected to a temperature ramp from 60 to $99^{\circ} \mathrm{C}$, rising $1{ }^{\circ} \mathrm{C}$ each step. For wild-type samples, single peaks were observed at $80^{\circ} \mathrm{C}$ while samples containing mutant alleles produced single peaks at $85^{\circ} \mathrm{C}$.

\section{Statistical Analysis}

The end points selected for analysis were overall survival defined as months from the date of initial diagnosis to the date of death or date of last followup and recurrence-free survival defined as months from the date of initial diagnosis to the date of first tumor recurrence, either metastatic or local. Outcomes were estimated by the Kaplan-Meier method and compared between the colorectal carcinoma age groups using the log-rank test. Univariate relationships between colorectal carcinoma age groups and continuous measures were assessed with Wilcoxon rank sum test and associations between colorectal carcinoma age groups and categorical measures were assessed with $\chi^{2}$ or Fisher's exact test. All statistics were assessed using two-sided tests with $P$-values $<0.05$ considered statistically significant. All statistical analyses were performed using SAS version 9.3 (SAS Institute, Cary, NC, USA).

\section{Results}

\section{Identification of Early-Onset and Control Group Colorectal Adenocarcinoma Groups}

A total of 1160 primary colorectal adenocarcinomas were surgically resected for the years 2000 through 2010. Of these, $75(6 \%)$ were diagnoses in patients $\leq 40$ years of age and were analyzed by either mismatch repair protein immunohistochemistry alone $(n=43)$ or both mismatch repair protein immunohistochemistry and microsatellite instability PCR (MSI PCR) analysis $(n=32)$ to select for cases with proficient DNA mismatch repair. Of the 75 colorectal carcinomas diagnosed in patients $\leq 40$ years of age, $13(17 \%)$ demonstrated abnormal mismatch repair protein expression, five with concurrent loss of MLH1 and PMS2 expression, five with isolated loss of MSH6 expression, two with concurrent loss of MSH2 and MSH6 expression, and one with isolated loss of PMS2 expression. In all, 5/ 13 cases with abnormal mismatch repair protein expression had concurrent MSI PCR with four cases demonstrating high levels of microsatellite instability and one case lacking microsatellite instability. Of the 75 colorectal carcinomas diagnosed in patients $\leq 40$ years of age, four additional patients had wellknown, documented germline genetic disorders predisposing to colorectal carcinoma: two patients familial adenomatous polyposis syndrome, one patient with juvenile polyposis syndrome, and one patient with Li-Fraumeni syndrome. Finally, three patients had a history of long-standing and histologically confirmed inflammatory bowel disease, two patients with ulcerative colitis and one patient with Crohn's disease. After excluding these patients with abnormal DNA mismatch repair $(n=13)$ and genetic or inflammatory conditions known to predispose to colorectal cancer $(n=7), 55$ remaining patients comprised the sporadic earlyonset colorectal carcinoma group, which was $5 \%$ of all colorectal carcinomas resected for the years 2000 through 2010.

Pathologic material was available for 85 consecutively resected colorectal carcinomas diagnosed in patients $>40$ years of age for the years 2005 and 2006. Of the 85 patients, $12(14 \%)$ demonstrated abnormal mismatch repair protein expression, 10 with concurrent loss of MLH1 and PMS2, one with isolated loss of MSH6, and one with isolated loss of PMS2. All patients with abnormal DNA mismatch repair $(n=12)$ were further excluded from the control group of colorectal carcinoma in patients $>40$ years of age which comprised a total of 73 patients. 

Clinicopathologic Features of Early-Onset and Control
Group Colorectal Adenocarcinomas

The clinicopathologic features of the early-onset and control colorectal carcinoma groups are detailed in Table 1. For the early-onset colorectal carcinoma group, the mean age was 34 years (range 14-40 years) with a slight male predominance (53\%), although this was not statistically significant compared with the control colorectal carcinoma group $(P=0.19)$. For the early-onset colorectal carcinoma group, most cases $(33 / 55,60 \%)$ were diagnosed between the age of 35 and 40 years of age, with fewer numbers diagnosed at $<20$ years $(2 / 55,4 \%), 20-29$ years $(7 / 55$, $13 \%)$, and $30-34$ years $(13 / 55,24 \%)$. Compared with the control group, the early-onset colorectal carcinoma group was significantly different with respect to tumor location $(P<0.007)$ with $80 \% \quad(44 / 55$ cases $)$ identified in either the sigmoid colon $(24 / 55,44 \%)$ or rectum $(20 / 55,36 \%)$. No difference was identified between the two groups with regard to tumor stage $(P=0.21)$, tumor size $(P=0.22)$, or number of lymph node metastases $(P=0.69)$. Morphologically, earlyonset colorectal carcinomas more frequently displayed adverse histologic features compared with the control colorectal carcinoma group such as signet ring cell differentiation $(7 / 55,13 \%$ vs $1 / 73,1 \%$, $P=0.021)$, perineural invasion $(16 / 55,29 \%$ vs $8 / 73$, $11 \%, P=0.009)$, and venous invasion $(12 / 55,22 \%$ vs $4 / 73,6 \%, P=0.006)$. Early-onset colorectal carcinomas were more frequently high grade $(15 / 55,27 \%)$ compared with the control group (10/73, 14\%) although this did not reach statistical significance $(P=0.06)$. The early-onset colorectal carcinoma group was less likely to be associated with a Crohn's-like inflammatory reaction $(12 / 55,22 \%)$ compared with the control group $(32 / 73,44 \%$, $P=0.024)$. There was no difference in the occurrence of lymphatic invasion, tumor budding, mucinous histology, or tumor-infiltrating lymphocytes between the early-onset and control colorectal carcinoma groups. Interestingly, a precursor adenomatous lesion was less frequently identified in the early-onset colorectal carcinoma group compared with the control group (19/55, 35\% vs 39/73, 53\%, $P=0.034)$. Only $4 / 55$ patients $(7 \%)$ had synchronous adenomatous polyps on presurgical colonoscopic evaluation or within the surgical resection specimen, and the number of polyps identified ranged from one to three.

\section{Molecular and Immunohistochemical Analysis of Early-Onset and Control Group Colorectal Adenocarcinomas}

Of the early-onset colorectal carcinomas, only two cases $(4 \%)$ demonstrated KRAS mutations, both in codon 12 of the KRAS gene, compared with 11/73 $(15 \%)$ of the control group colorectal adenocarcinomas harboring KRAS mutations, most frequently in codon 12 of the KRAS gene (10 cases), although this did not reach statistical significance $(P=0.13)$
Table 1 Clinicopathologic features of sporadic early-onset $(\leq 40$ years of age) colorectal and control ( $>40$ years of age) colorectal adenocarcinomas

\begin{tabular}{|c|c|c|c|}
\hline $\begin{array}{l}\text { Clinicopathologic } \\
\text { feature }\end{array}$ & $\begin{array}{c}\text { Early-onset } \\
\text { ( } \leq 40 \text { years) } \\
\text { colorectal } \\
\text { adenocarcinoma }\end{array}$ & $\begin{array}{c}\text { Control } \\
(>40 \text { years of age }) \\
\text { colorectal } \\
\text { adenocarcinomas }\end{array}$ & P-value \\
\hline Total number & 55 & 73 & - \\
\hline $\begin{array}{l}\text { Mean age, years } \\
\text { (range) }\end{array}$ & $34(14-40)$ & $65(41-95)$ & - \\
\hline \multicolumn{4}{|l|}{ Sex } \\
\hline Male & $29(53)$ & $30(41)$ & \multirow[t]{2}{*}{0.19} \\
\hline Female & $26(47)$ & $43(59)$ & \\
\hline \multicolumn{4}{|l|}{ Tumor location } \\
\hline Right colon & $11(20)$ & $31(42)$ & \multirow[t]{4}{*}{0.007} \\
\hline $\begin{array}{l}\text { Left colon and } \\
\text { rectum }\end{array}$ & $44(80)$ & $42(58)$ & \\
\hline Sigmoid & $24(44)$ & $33(45)$ & \\
\hline Rectum & $20(36)$ & $9(12)$ & \\
\hline \multicolumn{4}{|l|}{ Tumor stage } \\
\hline I & $6(11)$ & $14(19)$ & \multirow[t]{4}{*}{0.21} \\
\hline II & $14(26)$ & $21(29)$ & \\
\hline III & $20(36)$ & $28(38)$ & \\
\hline IV & $15(27)$ & $10(14)$ & \\
\hline $\begin{array}{l}\text { Median tumor size } \\
\text { (cm) (range) }\end{array}$ & $4.5(0.5-11.5)$ & $4.0(1.4-10.5)$ & 0.22 \\
\hline \multicolumn{4}{|l|}{ Tumor grade } \\
\hline Low & $40(73)$ & $63(86)$ & \multirow[t]{2}{*}{0.06} \\
\hline High & $15(27)$ & $10(14)$ & \\
\hline $\begin{array}{l}\text { Lymphatic } \\
\text { invasion }\end{array}$ & $25(46)$ & $27(37)$ & 0.33 \\
\hline \multicolumn{4}{|c|}{ Lymph node metastasis } \\
\hline 0 (No) & $22(42)$ & $37(51)$ & \multirow[t]{5}{*}{0.69} \\
\hline 1 (N1a) & $5(9)$ & $7(10)$ & \\
\hline $2-3$ (N1b) & $11(21)$ & $15(21)$ & \\
\hline 4-6 (N2a) & 7 (13) & $5(7)$ & \\
\hline$\geq 7$ (N2b) & $8(15)$ & $8(11)$ & \\
\hline $\begin{array}{l}\text { Perineural } \\
\text { invasion }\end{array}$ & $16(29)$ & $8(11)$ & 0.009 \\
\hline $\begin{array}{l}\text { Positive surgical } \\
\text { margins }\end{array}$ & $9(16)$ & $1(1)$ & 0.002 \\
\hline Venous invasion & $12(22)$ & $4(6)$ & 0.006 \\
\hline $\begin{array}{l}\text { Tumor-infiltrating } \\
\text { lymphocytes }\end{array}$ & $3(6)$ & $4(6)$ & 1.0 \\
\hline \multicolumn{4}{|l|}{ Crohn's-like reaction } \\
\hline Absent & $43(78)$ & $41(56)$ & \multirow[t]{3}{*}{0.024} \\
\hline Mild & $9(16)$ & $19(26)$ & \\
\hline Intense & $3(6)$ & $13(18)$ & \\
\hline \multicolumn{4}{|l|}{ Tumor budding } \\
\hline Low & $33(60)$ & $48(66)$ & \multirow[t]{2}{*}{0.50} \\
\hline High & $22(40)$ & $25(34)$ & \\
\hline \multicolumn{4}{|l|}{ Mucinous histology } \\
\hline Absent & $42(76)$ & $11(15)$ & \multirow[t]{2}{*}{0.22} \\
\hline Present & $13(24)$ & $62(85)$ & \\
\hline \multicolumn{4}{|l|}{ Signet ring histology } \\
\hline Absent & $7(13)$ & $1(1)$ & \multirow[t]{2}{*}{0.021} \\
\hline Present & $48(87)$ & 72 (99) & \\
\hline \multicolumn{4}{|c|}{ Precursor adenomatous lesion } \\
\hline Present & $19(35)$ & $39(53)$ & \multirow[t]{2}{*}{0.034} \\
\hline Absent & $36(66)$ & $34(47)$ & \\
\hline \multicolumn{4}{|c|}{ Precursor serrated lesion } \\
\hline Present & $4(7)$ & $10(14)$ & \multirow[t]{2}{*}{0.25} \\
\hline Absent & $51(93)$ & $63(86)$ & \\
\hline
\end{tabular}


Table 2 Immunohistochemical and molecular features of sporadic early-onset ( $\leq 40$ years of age) and control ( $>40$ years of age) colorectal adenocarcinomas

\begin{tabular}{|c|c|c|c|}
\hline $\begin{array}{l}\text { Immunohisto- } \\
\text { chemical or } \\
\text { molecular } \\
\text { feature }\end{array}$ & $\begin{array}{c}\text { Early-onset } \\
(\leq 40 \text { years }) \\
\text { colorectal } \\
\text { adenocarcinoma }(\%)\end{array}$ & $\begin{array}{c}\text { Control } \\
(>40 \text { years } \\
\text { of age) colorectal } \\
\text { adenocarcinoma }(\%)\end{array}$ & $\begin{array}{c}\mathrm{P}- \\
\text { value }\end{array}$ \\
\hline \multicolumn{4}{|c|}{ KRAS mutation } \\
\hline Positive & $2(4)$ & $11(15)$ & \multirow[t]{2}{*}{0.13} \\
\hline Negative & $43(96)$ & $62(85)$ & \\
\hline \multicolumn{4}{|c|}{ BRAF V600E mutation } \\
\hline Positive & 0 & $1(1)$ & \multirow[t]{2}{*}{1.0} \\
\hline Negative & $45(100)$ & $72(99)$ & \\
\hline \multicolumn{4}{|c|}{ Membranous E-cadherin expression } \\
\hline Present & $46(98)$ & $73(100)$ & \multirow[t]{2}{*}{0.39} \\
\hline Absent & $1(1)$ & 0 & \\
\hline \multicolumn{4}{|c|}{ Nuclear $\beta$-catenin expression } \\
\hline Present & $23(48)$ & $34(47)$ & \multirow[t]{2}{*}{0.88} \\
\hline Absent & $25(52)$ & $39(53)$ & \\
\hline \multicolumn{4}{|c|}{ Nuclear p16 expression } \\
\hline Present & $41(85)$ & $52(71)$ & \multirow[t]{2}{*}{0.07} \\
\hline Absent & $7(15)$ & $21(29)$ & \\
\hline \multicolumn{4}{|c|}{$H P V$ in-situ hybridization } \\
\hline Present & 0 & 0 & \multirow[t]{2}{*}{1.0} \\
\hline Absent & $48(100)$ & $73(100)$ & \\
\hline
\end{tabular}

(Table 2). BRAF V600E mutations were not identified in the early-onset colorectal carcinoma group and only one case in the control group was positive for the BRAF mutation. The early-onset colorectal adenocarcinomas more frequently displayed intact p16 expression $(41 / 55,85 \%)$ compared with the control group $(52 / 73,71 \%)$, although this did not reach statistical significance $(P=0.07)$ (Figure 1$)$. No differences in membranous E-cadherin or nuclear $\beta$-catenin expression were identified between the two groups. No tumor in either group demonstrated positive staining with HPV in-situ hybridization analysis.

\section{Clinical Outcome Stratified by Colorectal Carcinoma Age Group}

Three outcomes were assessed between the groups: recurrence, overall survival, and recurrence-free survival. Follow-up status was known with 54/55 patients in the early-onset colorectal carcinoma group and 63/73 patients in the control colorectal carcinoma group. An additional seven patients were never rendered free of their disease because they had surgical resection of the primary tumor with unresected metastatic disease. Therefore, recurrence and recurrence-free survival were evaluable for 110 patients and overall survival was evaluable for 117 patients. Both groups had similar recurrence-free $(P=0.28)$ and overall survival $(P=0.73)$ (Table 3 ; Figure 2). Patients in the early-onset colorectal carcinoma group had increased rates of recurrence (combined metastatic and local recurrence) compared with the control group of colorectal carcinoma (56 vs $36 \%$ at 5 years follow-up), although this did not reach statistical significance $(P=0.08)$ (Table 3 ; Figure 2). Patients in the early-onset colorectal carcinoma group more frequently either presented with or developed metastatic disease during their disease course compared with the control colorectal carcinoma group $(25 / 55,45 \%$ vs $18 / 73,25 \%$, $P=0.014)$. Patients in the early-onset colorectal carcinoma group more frequently had metastatic disease at presentation compared with the control colorectal carcinoma group (15/55, 27\% vs 10/73, $14 \%$ ), although this did not reach statistical significance $(P=0.06)$. Finally, $8 / 55$ patients $(15 \%)$ in the early-onset colorectal carcinoma developed local recurrence of their tumor while no patients in the control colorectal carcinoma group developed local recurrence $(P<0.001)$, likely due to the increased incidence of rectal carcinoma in the patients with early-onset colorectal carcinoma.

\section{Discussion}

In this study, early-onset colorectal carcinoma was defined as carcinoma presenting $\leq 40$ years of age. While the age cutoff employed in our analysis is somewhat arbitrary, the $\leq 40$ years of age group was selected because the most recent screening guidelines published by the American College of Gastroenterology recommend screening patients beginning at 50 years for the average-risk population or 45 years for African Americans. ${ }^{23}$ Our results demonstrate that colorectal carcinoma is not infrequently diagnosed in patients $\leq 40$ years of age, comprising $6 \%$ of all cases of colorectal carcinoma resected at our institution over a 10-year period, indicating that heightened clinical suspicion for colorectal carcinoma in young patients is warranted in order to ensure early diagnosis before presentation as advanced stage disease. Most tumors were diagnosed in fourth decade of life with a predominance occurring between the ages of 35 and 40 years of age. Surprisingly, in our analysis, most early-onset colorectal carcinomas did not appear to be Lynch syndrome associated, as only a small subset of cases demonstrated abnormalities in DNA mismatch repair. In addition, most early-onset colorectal carcinomas do not appear to be associated with other cancer-predisposing genetic conditions, such as colorectal polyposis syndromes, or chronic inflammatory conditions such as inflammatory bowel disease. We demonstrate that colorectal carcinomas identified in patients $\leq 40$ years of age have a striking predilection for the distal colon (80\%), particularly the sigmoid colon $(44 \%)$ and rectum $(36 \%)$. In addition, early-onset colorectal carcinomas were much more likely to demonstrate adverse histologic factors, including signet ring cell differentiation, venous invasion, and perineural invasion, and as a result are more likely to involve the 




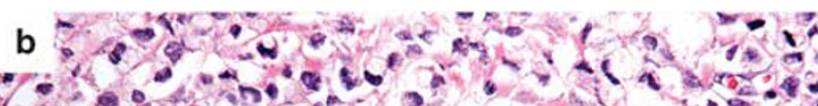

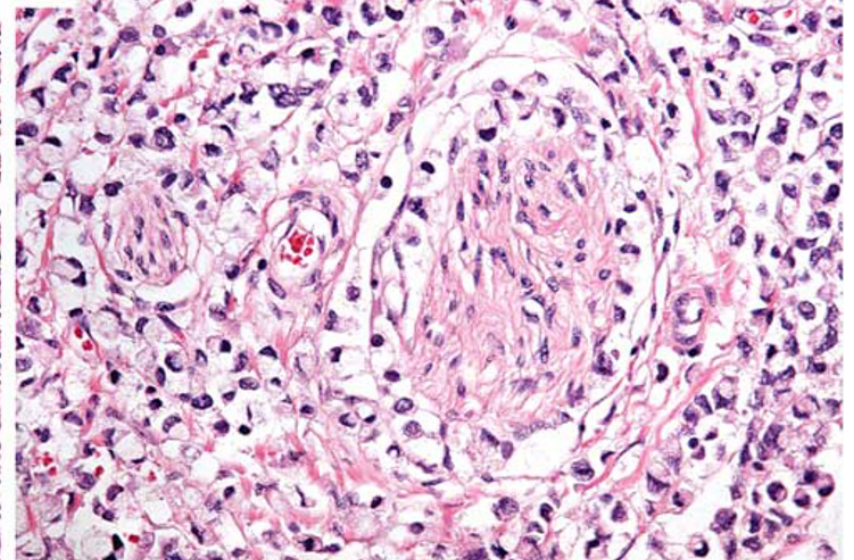
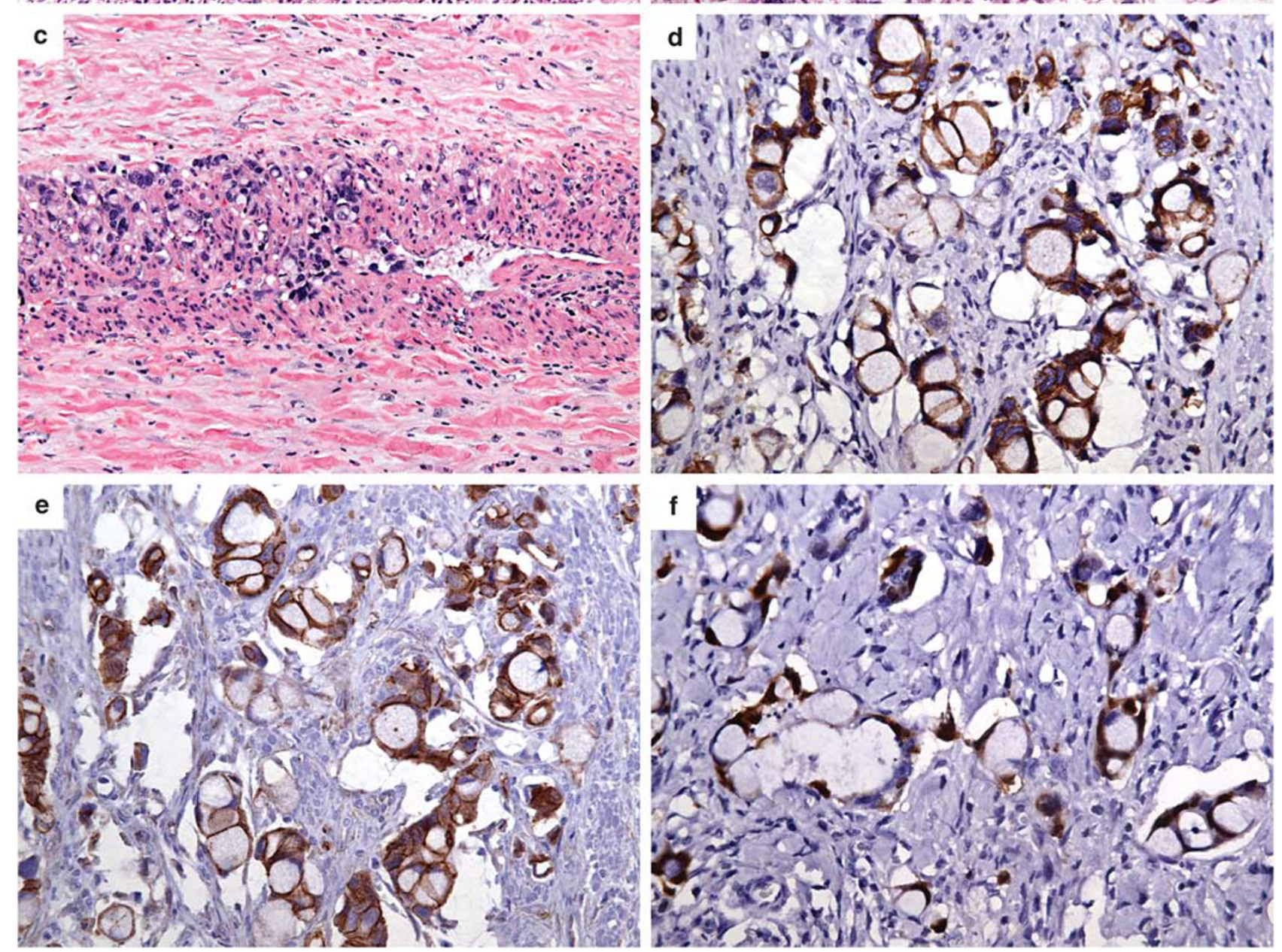

Figure 1 Early-onset rectal adenocarcinoma demonstrating signet ring histology without an associated precursor adenomatous lesion $(\mathbf{a}, \times 200)$. Early-onset colorectal adenocarcinomas often displayed extensive perineural invasion $(\mathbf{b}, \times 400)$, destructive venous invasion $(\mathbf{c}, \times 200)$, intact membranous E-cadherin expression $(\mathbf{d}, \times 400)$, lack of nuclear $\beta$-catenin localization $(\mathbf{e}, \times 400)$, and nuclear p16 expression $(\mathbf{f}, \times 400)$.

circumferential margins of surgical resection specimens. Our results also indicate that despite the presence of adverse histologic features, overall and recurrence-free survival in early-onset colorectal carcinoma is similar to patients $>40$ years of age, although a trend to increased incidence of recurrence was observed for early-onset colorectal carcinoma patients. Finally, early-onset colorectal carcinomas do not harbor BRAF V600E mutations and our results suggest that only a minor subset 
Table 3 Long-term follow-up according to colorectal carcinoma age group

\begin{tabular}{|c|c|c|c|c|c|c|c|c|c|c|c|c|c|}
\hline \multirow[t]{2}{*}{ Group } & \multirow[t]{2}{*}{ Patients } & \multirow[t]{2}{*}{$N E D(\%)$} & \multirow[t]{2}{*}{$A W D(\%)$} & \multirow[t]{2}{*}{$D O D(\%)$} & \multicolumn{3}{|c|}{$\begin{array}{l}\text { Recurrence } \\
(\%)\end{array}$} & \multicolumn{3}{|c|}{$\begin{array}{l}\text { Recurrence-free } \\
\text { survival (\%) }\end{array}$} & \multicolumn{3}{|c|}{$\begin{array}{c}\text { Overall } \\
\text { survival (\%) }\end{array}$} \\
\hline & & & & & $\begin{array}{c}1 \\
\text { year }\end{array}$ & $\begin{array}{c}3 \\
\text { years } \\
(\mathrm{P}=0.08\end{array}$ & $\begin{array}{c}5 \\
\text { years }\end{array}$ & $\begin{array}{c}1 \\
\text { year }\end{array}$ & $\begin{array}{c}3 \\
\text { years } \\
(\mathrm{P}=0.28)\end{array}$ & $\begin{array}{c}5 \\
\text { years }\end{array}$ & $\begin{array}{c}1 \\
\text { year }\end{array}$ & $\begin{array}{c}3 \\
\text { years } \\
(\mathrm{P}=0.73)\end{array}$ & $\begin{array}{c}5 \\
\text { years }\end{array}$ \\
\hline $\begin{array}{l}\text { Early-onset ( } \leq 40 \text { years) } \\
\text { colorectal adenocarcinoma }\end{array}$ & 54 & $25(46)$ & $13(24)$ & $16(30)$ & 19 & 52 & 56 & 76 & 45 & 35 & 94 & 80 & 53 \\
\hline $\begin{array}{l}\text { Control ( }>40 \text { years of age) } \\
\text { colorectal adenocarcinoma }\end{array}$ & 63 & $38(60)$ & $8(13)$ & $17(27)$ & 12 & 32 & 36 & 79 & 54 & 48 & 91 & 70 & 57 \\
\hline
\end{tabular}

Abbreviations: AWD, alive with disease; DOD, dead of disease; NED, alive with no evidence of disease.

(4\%) of these tumors display activating KRAS codon 12,13 , or 61 mutations.

Recent literature suggests an increasing incidence of colorectal carcinoma in young patients. ${ }^{2-4}$ Our analysis confirms observations in previous reports that colorectal carcinoma in young patients appears to occur more frequently in the rectum and rectosigmoid., ${ }^{2,3}$ Data from the SEER registry (19732005) identified an increasing annual percentage change of $2.2 \%$ of rectosigmoid cancer diagnosed in patients under the age of 40 years $^{2}$ with greatest percentage change in rectal carcinoma incidence was identified in patients between 35 and 39 years. ${ }^{2}$ Indeed, the 35-40 age group accounted for $60 \%$ of the early-onset colorectal carcinomas in our study. Our data and the data from the SEER registry indicate that rectal and rectosigmoid cancer in young patients is independent of gender. Earlier SEER data (1973-1999) also suggested that colorectal carcinoma in patients under the age of 40 years more frequently presented at advanced stage with distant metastasis than tumors in older patients $(27$ vs $19.7 \%, P<0.0001){ }^{3}$ A recent analysis by Yantiss et $a 1^{12}$ also demonstrated that up to $75 \%$ of earlyonset colorectal carcinoma presented with advanced (stages III and IV) disease. Our results confirm that patients with early-onset colorectal carcinoma more frequently develop metastatic disease during their disease course. Also, we identified a trend to increased incidence of metastatic disease at presentation for patients with early-onset colorectal carcinoma, although this did not reach statistical significance. In the SEER analysis (1973-2005), $2.6 \%$ of the colorectal carcinomas identified in patients $<40$ years of age demonstrated signet ring histology, ${ }^{2}$ which is lower than the $13 \%$ of tumors identified to have signet ring histology in our study. One reason may be that the SEER analysis included all tumor types, including squamous cell carcinoma and neuroendocrine tumors, which were specifically excluded from the current study. In addition, data from the SEER registry (1973-1999) also demonstrated that colorectal carcinoma in patients under the age of 40 years were more frequently poorly differentiated than tumors in older patients
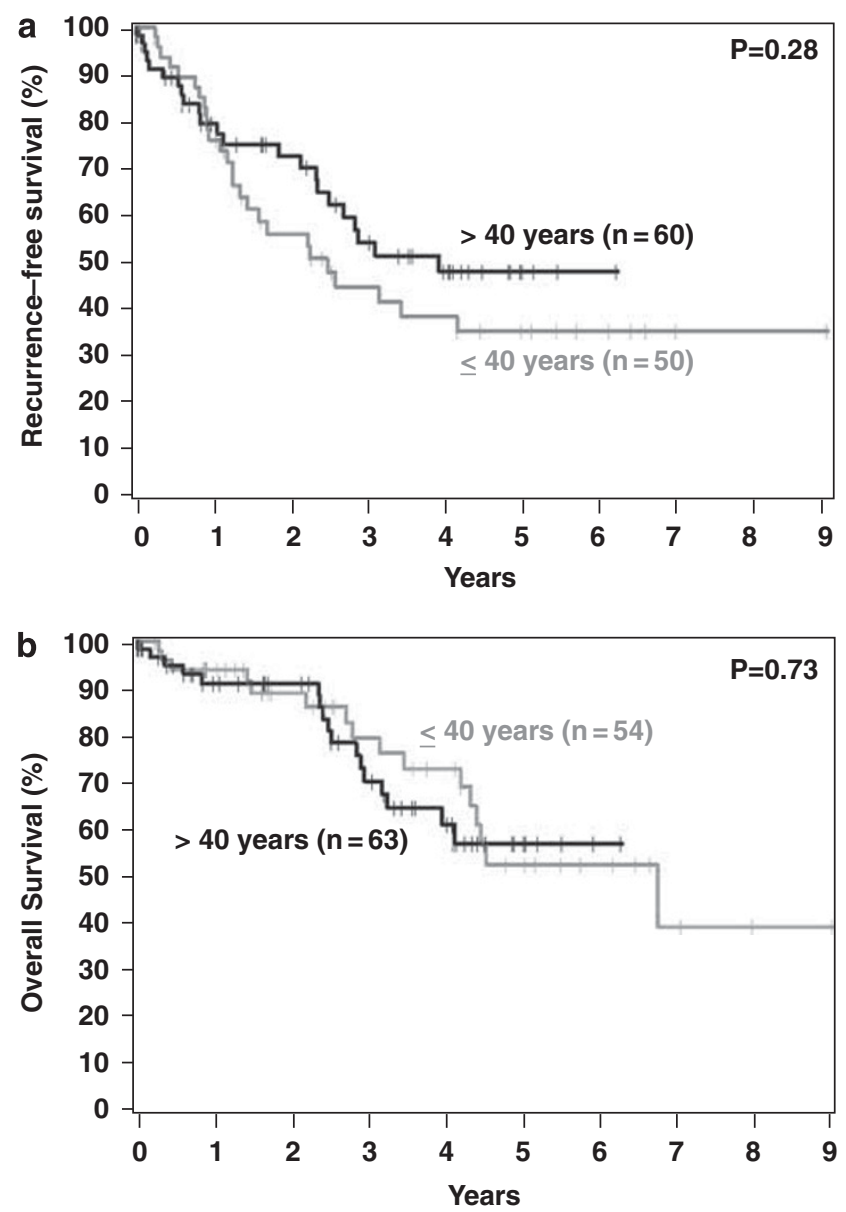

Figure 2 Kaplan-Meier survival curves comparing the recurrence-free (a) and overall survival (b) of patients with early-onset colorectal carcinoma ( $\leqslant 40$ years) and the control colorectal carcinoma group of patients $>40$ years of age. No significant difference in recurrence-free $(P=0.28)$ or overall survival $(P=0.73)$ was identified between the two groups.

(22.2 vs $14.7 \%, \quad P<0.0001){ }^{3}$ Our analysis also suggests that colorectal carcinoma in patients $\leq 40$ years of age are more frequently high grade, although this did not reach statistical significance $(P=0.06)$. Our results demonstrate that early-onset colorectal carcinomas are much more likely to 
demonstrate adverse histologic factors such as perineural, venous invasion, signet ring histology, and positive surgical margins. Similarly, Yantiss et al demonstrated that adverse histologic factors, including venous invasion and an infiltrating growth pattern, were typical of early-onset colorectal carcinomas, although in their analysis all tumors including those with deficient DNA mismatch repair or arising in the setting of inflammatory bowel disease were studied. These tumors were specifically excluded in our analysis.

This study expands on previous data and demonstrates that colorectal carcinoma in young patients is not always related to colorectal polyposis syndromes or Lynch syndrome, as only a small subset of colorectal carcinomas identified in patients $\leq 40$ years of age in our series were identified in patients with polyposis syndrome $(4 \%)$ or Lynch syndrome (17\%). Similarly, Goel et $a 2^{24}$ analyzed 75 colorectal carcinomas in patients under the age of 50 years and demonstrated that only $21 \%$ demonstrated abnormal mismatch protein repair expression by immunohistochemistry or high levels of microsatellite instability by PCR, suggesting a diagnosis of Lynch syndrome. Although the incidence of mismatch repair protein abnormalities and high levels of microsatellite instability suggesting a diagnosis of Lynch syndrome is relatively low (17\%) in colorectal carcinoma patients $\leq 40$ years of age, it is still much higher than the reported $3-5 \%$ incidence of Lynch syndrome in colorectal carcinoma identified in all age groups. Thus, routine analysis for mismatch repair protein abnormalities and microsatellite instability in young patients with colorectal carcinoma should be performed, as these Lynch syndrome patients and their family members are at risk for synchronous or metachronous Lynch syndrome-related tumors ${ }^{25}$ and would benefit from close surveillance and genetic counseling.

The limited literature on clinical outcomes in young patients with colorectal carcinoma is often confounded by the inclusion of inclusion of hereditary colorectal carcinoma, particularly Lynch syndrome-associated colorectal carcinomas, which likely have differing genetic events leading to tumorigenesis and often have improved survival compared with non-syndromic colorectal carcinoma. ${ }^{13,14}$ Our study is unique in that we specifically excluded potential Lynch syndrome patients from our survival analysis and demonstrate no significant overall or recurrence-free survival differences between patients with early-onset colorectal carcinoma and those patients with colorectal carcinoma $>40$ years of age. However, we did detect a trend to increased recurrence incidence in patients with colorectal carcinoma $\leq 40$ years of age. Numerous singleinstitution studies have demonstrated poor clinical outcomes for patients with early-onset colorectal carcinoma, ${ }^{5-9}$ although none of these studies specifically excluded Lynch syndrome patients from their survival analyses. A number of these studies ${ }^{5,6}$ found that patients with colorectal carcinoma $\leq 40$ years of age had increased incidence of colorectal carcinoma with mucinous histology, a morphologic finding that is often seen in association with Lynch syndrome. Other literature reports, including a large populationbased analysis with SEER data, have found that while young patients with colorectal carcinoma often present with advanced stage disease, these patients tend to have an equivalent or better cancer-specific survival rates compared with older patients. ${ }^{8,10}$ Again, these data are likely confounded by the inclusion of Lynch syndrome patients, which typically have improved survival compared with nonLynch syndrome-related colorectal carcinoma. The limited literature data are also conflicting with regards to the more common locations of colorectal carcinoma with some indicating increased frequency of proximal right-sided colorectal carcinoma ${ }^{11}$ while others demonstrating increased frequency of rectal and rectosigmoid carcinoma. ${ }^{3,12}$ Again, the discrepancy between these studies may be related to the proportion of Lynch syndrome-related colorectal carcinomas included in each study, as Lynch syndrome-related colorectal carcinomas frequently occur in the right colon. Our data clearly demonstrate a strong predilection for the left colon and rectum in sporadic early-onset colorectal carcinoma.

The molecular pathogenesis of colorectal carcinoma in young patients has not been well studied. Most colorectal carcinomas arise through a conventional adenoma-carcinoma sequence. ${ }^{26-28}$ Along the adenoma-carcinoma sequence, colorectal tumorigenesis can progress through either chromosomal instability pathway with multiple alterations in chromosome number, chromosomal rearrangements, or gene amplications or the microsatellite instability pathway as a result of a germiline mutation in a DNA mismatch repair protein. ${ }^{29}$ More recently, colorectal carcinomas that exhibit high levels of promoter methylation of $\mathrm{CpG}$ islands have been identified (CIMP-high, CIMP-H), ${ }^{30,31}$ and these CIMP-H carcinomas are thought to arise through a precursor sessile serrated polyp/adenoma. ${ }^{29} \mathrm{Ab}$ normalities of the Wnt signaling pathway, particularly APC inactivation with nuclear localization of $\beta$-catenin, and activating KRAS mutations are common events in the adenoma-carcinoma sequence of the chromosomal instability pathway. Wnt signaling pathway abnormalities, particularly abnormalities of $\beta$-catenin, also likely have a role in CIMP-high colorectal carcinomas, as abnormal nuclear localization of $\beta$-catenin is frequently identified in sessile serrated polyps/adenomas. ${ }^{32,33}$ Importantly, $\sim 60-80 \%$ of CIMP-H tumors harbor mutations in BRAF, and CIMP-H tumors rarely, if ever, demonstrate KRAS mutations. ${ }^{31,34}$ In addition, CIMP-H colorectal carcinomas often demonstrate methylation of the $p 16$ promoter, resulting in diminished or absent p16 protein expression. In our analysis, very few of the colorectal carcinomas demonstrated BRAF V600E mutations, indicating 
that the promoter methylation of $\mathrm{CpG}$ islands (CIMP-H) is not important in the neoplastic progression of colorectal carcinoma in young patients. In addition, most early-onset colorectal carcinomas demonstrated intact nuclear p16 expression, providing further support that promoter methylation of CpG islands is not a critical molecular event in colorectal carcinoma in patients $\leq 40$ years of age. Only two cases of early-onset colorectal carcinoma in our series were associated with a precursor serrated lesion, both of which were traditional serrated adenomas; no cases were associated with sessile serrated polyp/adenoma.

In our analysis, only a minority $(4 \%)$ of early-onset colorectal carcinomas demonstrated activating KRAS codon 12, 13, or 61 mutations. Goel et $a l^{24}$ analyzed 75 colorectal carcinomas diagnosed in patients under the age of 50 and identified 59 with proficient mismatch repair. Of these 59 patients, most were in the distal colorectum $(42 / 59,71 \%)$, none demonstrated BRAF mutations, and 14/59 (24\%) demonstrated KRAS mutations. ${ }^{24}$ Our results identified a lower rate of KRAS mutation (4\%) in early-onset colorectal carcinoma. The reason for this discrepancy is not entirely clear, although a lower frequency of KRAS mutation was also observed in our control group of carcinomas identified over the age of 40 years. Interestingly, in our analysis early-onset colorectal carcinomas were less frequently associated with a precursor adenomatous lesion compared with the older age group control population. It is possible that the conventional colorectal adenoma-carcinoma sequence, which typically results in early KRAS mutation in the pathway to carcinogenesis, may not apply to the young patient population. However, the reduced number of precursor adenomatous lesions identified in early-onset colorectal carcinoma in our study may be the result of surgical resections of smaller length performed on these patients given the increased incidence of carcinoma involving the rectum and sigmoid. In addition, nearly half $(48 \%)$ of colorectal carcinomas in patients $\leq 40$ years of age demonstrated nuclear $\beta$-catenin expression, suggesting that abnormalities in the Wnt signaling pathway are still important for tumor development in young colorectal cancer patients similar to colorectal carcinoma in older patients. The molecular evolution of colorectal carcinogenesis in the young patient population requires further study.

The role of human papillomavirus in colorectal carcinoma remains controversial. Some authors have demonstrated HPV DNA in 46/72 (64\%) ${ }^{35}$ and 23/55 $(42 \%)^{36}$ of colorectal carcinoma, suggesting a role for HPV infection, particularly HPV type 16, in colorectal carcinogenesis. However, both of these studies also reported the presence of HPV DNA in a large proportion of histologically normal mucosa adjacent to the colorectal carcinomas. Our analysis clearly demonstrates the absence of HPV DNA in colorectal carcinoma tumor cells, as our in-situ hybridization methods allows for detection of HPV DNA specifi- cally within tumor cell nuclei. Similarly, Yantiss et $a^{12}$ did not identify HPV DNA in any of their cases of early-onset colorectal carcinoma.

Finally, the incidence of signet ring differentiation in early-onset colorectal carcinomas in our analysis is intriguing. Of the six signet ring carcinomas identified in patients $\leq 40$ years of age, all six demonstrated intact membranous E-cadherin expression and lacked nuclear $\beta$-catenin expression. Typically, hereditary signet ring carcinoma observed in the stomach of patients with germline $C D H 1$ mutation demonstrate diminished or absent E-cadherin with abnormal nuclear localization of $\beta$-catenin, although deeply invasive tumors may not display this immunophenotype. ${ }^{37}$ Our results suggest that germline $\mathrm{CDH} 1$ mutations are not likely in early-onset signet ring colorectal carcinomas and that alternative molecular events give rise to this tumor histology in young patients. In their analysis, Ogino et a ${ }^{38}$ analyzed 32 colorectal carcinomas with a signet ring component with $9(28 \%)$ demonstrating $B R A F$ mutations and $10(26 \%)$ demonstrating KRAS mutations, indicating that mutations in the EGFR signaling pathway are not infrequent in signet ring colorectal carcinoma. In our analysis, none of the signet ring carcinomas demonstrated BRAF or KRAS mutations. Importantly, Ogino et al included carcinomas with high levels of microsatellite instability, making comparison between our results difficult. Kakar and Smyrk ${ }^{39}$ studied 72 signet ring colorectal carcinomas of which 39 were right-sided with most right-sided carcinomas (81\%) exhibiting MSI-H and often seen in patients over the age of 70 years. In contrast, of the MSS signet ring carcinoma in the analysis by Kakar and Smyrk, most were identified in patients under the age of 70 years and were located in the left colon, similar to our results. Interestingly, Kakar and Smyrk ${ }^{39}$ demonstrated no survival difference between MSS and unstable signet ring colorectal carcinomas. Overall, our results indicate that MSS signet ring carcinomas are typically identified in young patients, are more often located in the left colon and rectum, and do not necessarily confer reduced overall and recurrence-free survival.

After exclusion of potential Lynch syndrome patients and patients with known cancer-predisposing genetic or inflammatory conditions, we have labeled the early-onset colorectal carcinoma identified in our study as being sporadic. However, we cannot entirely exclude the possibility of familial colorectal carcinoma syndromes such as attenuated familial adenomatous polyposis and MUTYH-associated colorectal carcinoma, which typically do not manifest with innumerable colorectal polyps. Of the early-onset colorectal carcinomas included in our study, most were not associated with synchronous colorectal adenomatous or serrated polyps and of those with synchronous adenomatous or serrated polyps, the number of polyps identified ranged from one to three. Given the limited number of adenomatous or serrated polyps associated with the tumors 
identified in our study, it is unlikely that these patients have a familial polyposis colorectal carcinoma syndrome.

In conclusion, our study demonstrates that colorectal carcinoma is not infrequently diagnosed in patients $\leq 40$ years of age and are not frequently the result of underlying Lynch syndrome or associated with other cancer-predisposing genetic conditions, such as colorectal polyposis syndromes, or chronic inflammatory conditions. These tumors have a striking predilection for the distal colon (80\%), particularly the sigmoid colon and rectum and are much more likely to demonstrate adverse histologic factors, including signet ring cell differentiation, venous invasion, and perineural invasion. However, overall and recurrence-free survival in early-onset colorectal carcinoma is similar to patients $>40$ years of age. Early-onset colorectal carcinomas are not frequently associated with precursor adenomatous or serrated lesions and do not appear to harbor frequent activating $B R A F$ or $K R A S$ mutations, suggesting that the molecular events in tumor development differ in this patient population. Further molecular analysis is necessary to fully elucidate the potential molecular changes that lead to early-onset colorectal carcinoma.

\section{Disclosure/conflict of interest}

The authors declare no conflict of interest.

\section{References}

1 Jemal A, Siegel R, Xu J, et al. Cancer statistics, 2010. CA Cancer J Clin 2010;60:277-300.

2 Meyer JE, Narang T, Schnoll-Sussman FH, et al. Increasing incidence of rectal cancer in patients aged younger than 40 years: an analysis of the surveillance, epidemiology, and end results database. Cancer 2010; 116:4354-4359.

3 O’Connell JB, Maggard MA, Liu JH, et al. Rates of colon and rectal cancers are increasing in young adults. Am Surg 2003;69:866-872.

4 Pignone M, Rich M, Teutsch SM, et al. Screening for colorectal cancer in adults at average risk: a summary of the evidence for the US. Preventive Services Task Force. Ann Intern Med 2002;137:132-141.

5 D'Onofrio GM, Tan EG. Is colorectal carcinoma in the young a more deadly disease? Aust N Z J Surg 1985;55:537-540.

6 Mills SE, Allen Jr MS. Colorectal carcinoma in the first three decades of life. Am J Surg Pathol 1979;3:443-448.

7 Minardi Jr AJ, Sittig KM, Zibari GB, et al. Colorectal cancer in the young patient. Am Surg 1998;64:849-853.

8 Parramore JB, Wei JP, Yeh KA. Colorectal cancer in patients under forty: presentation and outcome. Am Surg 1998;64:563-567.

9 Smith C, Butler JA. Colorectal cancer in patients younger than 40 years of age. Dis Colon Rectum 1989;32:843-846.

10 O’Connell JB, Maggard MA, Liu JH, et al. Do young colon cancer patients have worse outcomes? World J Surg 2004;28:558-562.
11 Savas N, Dagli U, Akbulut S, et al. Colorectal cancer localization in young patients: should we expand the screening program? Dig Dis Sci 2007;52:798-802.

12 Yantiss RK, Goodarzi M, Zhou XK, et al. Clinical, pathologic, and molecular features of early-onset colorectal carcinoma. Am J Surg Pathol 2009;33:572-582.

13 Popat S, Hubner R, Houlston RS. Systematic review of microsatellite instability and colorectal cancer prognosis. J Clin Oncol 2005;23:609-618.

14 Bertagnolli MM, Niedzwiecki D, Compton CC, et al. Microsatellite instability predicts improved response to adjuvant therapy with irinotecan, fluorouracil, and leucovorin in stage III colon cancer: Cancer and Leukemia Group B Protocol 89803. J Clin Oncol 2009; 27:1814-1821.

15 Wang LM, Kevans D, Mulcahy H, et al. Tumor budding is a strong and reproducible prognostic marker in T3No colorectal cancer. Am J Surg Pathol 2009;33: 134-141.

16 Smyrk TC, Watson P, Kaul K, et al. Tumor-infiltrating lymphocytes are a marker for microsatellite instability in colorectal carcinoma. Cancer 2001;91:2417-2422.

17 Graham DM, Appelman HD. Crohn's-like lymphoid reaction and colorectal carcinoma: a potential histologic prognosticator. Mod Pathol 1990;3:332-335.

18 Hamilton SR, Bosman FT, Boffetta P, et al. Carcinoma of the colon and rectum. In: Bosman FT, Carneiro F, Hruban RH, Theise ND (eds). WHO Classification of Tumors of the Digestive System. IARC Press: Lyon, France, 2010, pp 134-146.

19 Snover DC, Ahnen DJ, Burt RW, et al. Serrated polyps of the colon and rectum and serrated polyposis. In: Bosman FT, Carneiro F, Hruban RH, Theise ND (eds). WHO Classification of Tumors of the Digestive System. IARC Press: Lyon, France, 2010, pp 160-165.

20 Suraweera N, Duval A, Reperant M, et al. Evaluation of tumor microsatellite instability using five quasimonomorphic mononucleotide repeats and pentaplex PCR. Gastroenterology 2002;123:1804-1811.

21 Jass JR, Baker K, Zlobec I, et al. Advanced colorectal polyps with the molecular and morphological features of serrated polyps and adenomas: concept of a 'fusion' pathway to colorectal cancer. Histopathology 2006;49: 121-131.

22 Young J, Barker MA, Simms LA, et al. Evidence for BRAF mutation and variable levels of microsatellite instability in a syndrome of familial colorectal cancer. Clin Gastroenterol Hepatol 2005;3:254-263.

23 Rex DK, Johnson DA, Anderson JC, et al. American college of gastroenterology guidelines for colorectal cancer screening 2009. Am J Gastroenterol 2009;104: 739-750.

24 Goel A, Nagasaka T, Spiegel J, et al. Low frequency of Lynch syndrome among young patients with nonfamilial colorectal cancer. Clin Gastroenterol Hepatol 2010;8:966-971.

25 Aarnio M, Sankila R, Pukkala E, et al. Cancer risk in mutation carriers of DNA-mismatch-repair genes. Int J Cancer 1999;81:214-218.

26 Fearon ER, Vogelstein B. A genetic model for colorectal tumorigenesis. Cell 1990;61:759-767.

27 Bos JL, Fearon ER, Hamilton SR, et al. Prevalence of ras gene mutations in human colorectal cancers. Nature 1987;327:293-297.

28 Vogelstein B, Fearon ER, Hamilton SR, et al. Genetic alterations during colorectal-tumor development. N Engl J Med 1988;319:525-532. 
29 Jass JR. Classification of colorectal cancer based on correlation of clinical, morphological and molecular features. Histopathology 2007;50:113-130.

30 Barault L, Charon-Barra C, Jooste V, et al. Hypermethylator phenotype in sporadic colon cancer: study on a population-based series of 582 cases. Cancer Res 2008;68:8541-8546.

31 Ogino S, Nosho K, Kirkner GJ, et al. CpG island methylator phenotype, microsatellite instability, BRAF mutation and clinical outcome in colon cancer. Gut 2009;58:90-96.

$32 \mathrm{Wu}$ JM, Montgomery EA, Iacobuzio-Donahue CA. Frequent beta-catenin nuclear labeling in sessile serrated polyps of the colorectum with neoplastic potential. Am J Clin Pathol 2008;129:416-423.

33 Yachida S, Mudali S, Martin SA, et al. Beta-catenin nuclear labeling is a common feature of sessile serrated adenomas and correlates with early neoplastic progression after BRAF activation. Am J Surg Pathol 2009; 33:1823-1832.

34 Nagasaka T, Koi M, Kloor M, et al. Mutations in both KRAS and BRAF may contribute to the methylator phenotype in colon cancer. Gastroenterology 2008;134:1950-1960.

35 Damin DC, Caetano MB, Rosito MA, et al. Evidence for an association of human papillomavirus infection and colorectal cancer. Eur J Surg Oncol 2007;33: 569-574.

36 Bodaghi S, Yamanegi K, Xiao SY, et al. Colorectal papillomavirus infection in patients with colorectal cancer. Clin Cancer Res 2005;11:2862-2867.

37 Rogers WM, Dobo E, Norton JA, et al. Risk-reducing total gastrectomy for germline mutations in E-cadherin (CDH1): pathologic findings with clinical implications. Am J Surg Pathol 2008;32:799-809.

38 Ogino S, Brahmandam M, Cantor M, et al. Distinct molecular features of colorectal carcinoma with signet ring cell component and colorectal carcinoma with mucinous component. Mod Pathol 2006;19: 59-68.

39 Kakar S, Smyrk TC. Signet ring cell carcinoma of the colorectum: correlations between microsatellite instability, clinicopathologic features and survival. Mod Pathol 2005;18:244-249. 